\title{
Dynamics and bifurcation near the transition from stability to complex instability
}

\author{
M. Ollé, J. R. Pacha and J. Villanueva \\ Dept. de Matemàtica Aplicada I, ETSEIB (UPC), Diagonal 647, \\ 08028 Barcelona, Spain \\ E-mail: olle@ma1.upc.es, joanr@vilma.upc.es and jordi@vilma.upc.es
}

\begin{abstract}
We consider a Hamiltonian of three degrees of freedom and a family of periodic orbits with a transition from stability to complex instability, such that there is an irrational collision of the Floquet mulipliers of opposite sign. We analyze the local dynamics and the bifurcation phenomena linked to this transition. We study the resulting Hamiltonian Hopf-like bifurcation from an analytical point of view by means of normal forms. The existence of a bifurcating family of $2 \mathrm{D}$ tori is derived and both cases (direct and inverse bifurcation) are described.
\end{abstract}

Key Words: normal forms, bifurcation, invariant tori

\section{INTRODUCTION}

Let us consider a Hamiltonian with three degrees of freedom and a family of periodic orbits with a transition from stability to complex instability. That is, we assume there is a periodic orbit for which two pairs of characteristic multipliers collide on the unit circle at an irrational point (i.e. the multipliers are $1,1, \lambda=\mu, 1 / \lambda=1 / \mu$ and $\lambda=\exp (i \theta), \theta=2 \pi \rho$ with $\rho$ irrational). From now on we call such orbit a critical periodic orbit with irrational collision (CPO).

Actually, complex instability is a typical phenomenon of periodic orbits in Hamiltonian systems with three or more degrees of freedom, and several papers have been devoted to describe it. From a numerical point of view, some studies have been done both for 4D symplectic mappings (see [4], [8] and [13]) and for some particular applications in celestial and galactic dynamics context (see for example [5], [9], [10], [14], [15] and [11] -and references therein-) 
Concerning analytical studies, Van der Meer ([16] described the originally called Hamiltonian Hopf bifurcation. Such bifurcation refers to a Hamiltonian with two degrees of freedom having a family of equilibrium points with a transition from stability to complex instability and bifurcating periodic orbits. When a Hamiltonian with three degrees of freedom and a critical periodic orbit with irrational collision are considered, a Hopf-like bifurcation takes place but with bifurcating 2-dimensional invariant tori: see [6], where the author uses the Hamiltonian itself but with a previous isoenergetic reduction and a normal form up to order six; and [3], where they consider a nonlinear 4D symplectic map, and use the normal form derived in [2] in order to simplify the map. We remark that the theory for the existence and stability of periodic orbits in the unfolding of a rational collision is analyzed in [1].

Our contribution in this paper is to present a constructive method (that can be implemented numerically, from a practical point of view) to compute the normal form around a critical periodic orbit with irrational collision, up to an arbitrary high order. The truncated normal form allows to obtain a good approximation of the relevant invariant objects (periodic orbits, 2D and 3D tori and invariant manifolds) that describe the dynamics close to the critical orbit.

Finally we remark that, along this paper, we will focus our attention on giving the main ideas about this problem and we refer the interested reader to [12] for the details and proofs.

\section{FORMULATION OF THE PROBLEM AND METHODOLOGY}

Let $H(\zeta)$ with $\zeta=(\xi, \eta)$, be a real three degree of freedom analytic Hamiltonian, with its associated Hamiltonian system

$$
\dot{\zeta}=J_{3} \nabla H(\zeta)
$$

where $J_{3}$ is the matrix of the standard canonical 2 -form of $R^{6}$ and $\dot{\zeta}=$ $d \zeta / d t$. We assume that this Hamiltonian system has a non degenerate family of periodic orbits with a transition from stability to complex instability, that is, there exists a CPO. we denote its period by $T_{0}=2 \pi / \omega_{0}$ and by $\zeta_{0}(\theta)$ a $2 \pi$-periodic parametrization of this critical orbit.

Our analytical approach is twofold: on one hand, we compute the (formal) normal form, around the critical periodic orbit, up to an arbitrarily high order. For this computation, we carry out the following process:

- We change the system of coordinates to a suitable one, by means of a symplectic transformation; 
- we apply a canonical Floquet transformation to reduce the normal variational equations of the orbit to constant coefficients;

- we complexify the Hamiltonian;

- we describe how to compute, in a some tricky and constructive way, the normal form.

On the other hand, we deal with the truncated normal form itself and the differential equations associated to it (we call them truncated $O D E$ ), in order to describe the dynamics near the transition. More precisely,

- we derive the existence of two families of invariant 2D tori which bifurcate from the critical orbit,

- we identify the coefficient that determines the unfolding type and the stability of the bifurcating 2D-tori. We obtain again a classical Hopf bifurcation: stable 2D-tori for the direct case and unstable bifurcating 2D-tori for the inverse case.

- We describe the evolution of the 2D-tori when varying the two integrals of the truncated ODE.

In the following section we present the explicit version of these results.

\section{NORMAL FORM PROCESS}

\subsection{The Jordan structure of the monodromy matrix}

Let us consider the critical periodic orbit (CPO) of period $T_{0}$, the $2 \pi$ parametrization $\zeta_{0}(\theta)$ and and the monodromy matrix (fundamental solution -after one period- of the variational differential system) $\mathbf{M}_{0}(2 \pi)$. Its Jordan normal form will have a double eigenvalue equal to 1 forming a nontrivial Jordan block (in the generic non degenerate sense), and two double complex eigenvalues $\lambda \neq \pm 1,1 / \lambda=\bar{\lambda}$ again in a two nontrivial blocks. Thus we have the following

Proposition 1. (i) There exists a symplectic complex basis $\left\{u_{1}, u_{2}, v_{1}, v_{2}\right.$, $\left.w_{1}, w_{2}\right\}$, with respect to the canonical 2-form, such that

1.

$$
\left.\mathbf{M}_{0}(2 \pi)\right|_{\left.<u_{1}, u_{2}, v_{1}, v_{2}, w_{1}, w_{2}\right\rangle}=\left(\begin{array}{cc|c|cc}
1 & a & & & \\
0 & 1 & & & \\
& & \lambda & \lambda \\
& 0 & \lambda & & \\
& & & 1 / \lambda & 0 \\
& & & -1 / \lambda & 1 / \lambda
\end{array}\right)
$$


2.If we define

$$
\mathbf{B}=\left(\begin{array}{cc|cc}
i \frac{\widetilde{\omega}_{1}}{T_{0}} & \frac{1}{T_{0}} & & \\
0 & i \frac{\omega_{1}}{T_{0}} & & \\
& & -i \frac{\widetilde{\omega}_{1}}{T_{0}} & 0 \\
-\frac{1}{T_{0}} & -i \frac{\widetilde{\omega}_{1}}{T_{0}}
\end{array}\right),
$$

with $\lambda=e^{-i \widetilde{\omega}_{1} / T_{0}}, i=\sqrt{-1}$, then $\mathbf{B}$ verifies $\left.\mathbf{M}_{0}(2 \pi)\right|_{\left\langle v_{1}, v_{2}, w_{1}, w_{2}>\right.}=e^{\mathbf{B} T_{0}}$, $i$. e. $\mathbf{B}$ is the Floquet matrix of the normal variational equations.

(ii) Let us define

$$
v_{1}^{*}=\frac{v_{1}-w_{2}}{\sqrt{2}}, \quad v_{2}^{*}=\frac{v_{1}+w_{2}}{\sqrt{2} i}, \quad w_{1}^{*}=\frac{v_{2}+w_{1}}{\sqrt{2}}, \quad w_{2}^{*}=\frac{v_{2}-w_{1}}{\sqrt{2} i}
$$

Then we have that $\left\{u_{1}, u_{2}, v_{1}^{*}, v_{2}^{*}, w_{1}^{*}, w_{2}^{*}\right\}$ is a symplectic real basis that reduces $\mathbf{M}_{0}(2 \pi)$ to its "real Jordan" normal form

$$
\left.\mathbf{M}_{0}(2 \pi)\right|_{<u_{1,2}, v_{1,2}^{*}, w_{1,2}^{*}>}=\left(\begin{array}{cc|cccc}
1 & a & & & & \\
0 & 1 & & & & \\
\hline & \cos \widetilde{\omega}_{1} & \sin \widetilde{\omega}_{1} & \cos \widetilde{\omega}_{1} & \sin \widetilde{\omega}_{1} \\
& -\sin \widetilde{\omega}_{1} & \cos \widetilde{\omega}_{1} & -\sin \widetilde{\omega}_{1} & \cos \widetilde{\omega}_{1} \\
& 0 & 0 & \cos \widetilde{\omega}_{1} & \sin \widetilde{\omega}_{1} \\
& 0 & 0 & -\sin \widetilde{\omega}_{1} & \cos \widetilde{\omega}_{1}
\end{array}\right)
$$

Moreover, in this basis the matrix $\mathbf{B}$ becomes:

$$
\mathbf{B}=\left(\begin{array}{cc|cc}
0 & \frac{\widetilde{\omega}_{1}}{T_{0}} & \frac{1}{T_{0}} & 0 \\
-\frac{\widetilde{\omega}_{1}}{T_{0}} & 0 & 0 & \frac{1}{T_{0}} \\
\hline 0 & 0 & 0 & \frac{\widetilde{\omega}_{1}}{T_{0}} \\
0 & 0 & -\frac{\widetilde{\omega}_{1}}{T_{0}} & 0
\end{array}\right)
$$

\subsection{The quadratic part of the Hamiltonian in the adapted coordinates}

Before starting the normal form process, it is convenient to perform some canonical changes of coordinates in order to have a suitable expression of the quadratic terms of the Hamiltonian. So, we assume that we can introduce a suitable system of canonical coordinates $([7])$ in order to translate the CPO to the origin. This adapted system of coordinates has to contain an angular variable $\theta$ to parametrize the whole periodic orbit, and its canonical conjugate variable $I$ (that parametrizes the family of periodic 
orbits). Moreover, we have to introduce other four Cartesian coordinates to describe the normal behavior of the orbit. As we have shown in the previous section, these normal coordinates can be chosen (real or complex) in such a way that the normal variational equations of the orbit are reduced to a constant coefficient system with matrix B. Let us remark that as it has been done in [7], these coordinates can be found explicitly, in some cases, in a practical implementation of this normal form methodology.

We denote by $(q, p)=\left(q_{1}, q_{2}, p_{1}, p_{2}\right)$ the complex normal variables introduced before, and we use the following notation: given an analytic function $f\left(\theta, q_{1}, q_{2}, I, p_{1}, p_{2}\right)$, we expand it as

$$
f\left(\theta, q_{1}, q_{2}, I, p_{1}, p_{2}\right)=\sum_{l, m_{1}, m_{2}, n_{1}, n_{2}} f_{l, m_{1}, m_{2}, n_{1}, n_{2}}(\theta) I^{l} q_{1}^{m_{1}} q_{2}^{m_{2}} p_{1}^{n_{1}} p_{2}^{n_{2}},
$$

with $l, m_{1}, m_{2}, n_{1}, n_{2} \in N$ and where $f_{l, m_{1}, m_{2}, n_{1}, n_{2}}(\theta)$ are analytic and $2 \pi$ functions, that may also be expanded as

$$
f_{l, m_{1}, m_{2}, n_{1}, n_{2}}(\theta)=\sum_{k \in Z} f_{l, m_{1}, m_{2}, n_{1}, n_{2}, k} \exp (i k \theta)
$$

We also introduce the following definition of degree for a given monomial $f_{l, m_{1}, m_{2}, n_{1}, n_{2}}(\theta) I^{l} q_{1}^{m_{1}} q_{2}^{m_{2}} p_{1}^{n_{1}} p_{2}^{n_{2}}$,

$$
\operatorname{deg}\left(I^{l} q_{1}^{m_{1}} q_{2}^{m_{2}} p_{1}^{n_{1}} p_{2}^{n_{2}}\right)=2 l+m_{1}+m_{2}+n_{1}+n_{2},
$$

we have that, in the $(\theta, q, I, p)$ variables $((q, p)$ suitably scaled $)$, the quadratic part of the Hamiltonian takes the form:

$$
H_{2}\left(\theta, q_{1}, q_{2}, I, p_{1}, p_{2}\right)=\omega_{0} I+i \omega_{1}\left(q_{1} p_{1}+q_{2} p_{2}\right)+q_{2} p_{1},
$$

where $\omega_{1}=\widetilde{\omega}_{1} / T_{0}$ and the CPO is given by $I=0, q=p=0$.

If in equation (7) we use the real Floquet variables $\left(x_{1}, x_{2}, y_{1}, y_{2}\right)$, obtained from the change of basis given in (4), then the quadratic part of the Hamiltonian becomes

$$
H_{2}\left(\theta, x_{1}, x_{2}, I, p_{1}, p_{2}\right)=\omega_{0} I+\omega_{1}\left(x_{1} y_{2}-x_{2} y_{1}\right)+\frac{1}{2}\left(y_{1}^{2}+y_{2}^{2}\right),
$$

where the variables $\left(x_{1}, x_{2}, y_{1}, y_{2}\right)$ are related with the complex ones, $\left(q_{1}, q_{2}, p_{1}, p_{2}\right)$ by

$$
q_{1}=\frac{x_{1}-i x_{2}}{\sqrt{2}}, \quad q_{2}=\frac{y_{1}-i y_{2}}{\sqrt{2}}, \quad p_{1}=\frac{y_{1}+i y_{2}}{\sqrt{2}}, \quad p_{2}=-\frac{x_{1}+i x_{2}}{\sqrt{2}},
$$

relations which follow immediately from (4). In particular,

$$
\bar{q}_{1}=-p_{2}, \quad \bar{p}_{1}=q_{2}, \quad \bar{q}_{2}=p_{1}, \quad \bar{p}_{2}=-q_{1} .
$$


The symmetries above imply that if we consider the following expansion of the Hamiltonian,

$$
H\left(\theta, q_{1}, q_{2}, I, p_{1}, p_{2}\right)=\sum_{l, m_{1}, m_{2}, n_{1}, n_{2}, k} h_{l, m_{1}, m_{2}, n_{1}, n_{2}, k} e^{i k \theta} I^{l} q_{1}^{m_{1}} q_{2}^{m_{2}} p_{1}^{n_{1}} p_{2}^{n_{2}},
$$

then we have the following symmetries coming from the complexification

$$
\bar{h}_{l, m_{1}, m_{2}, n_{1}, n_{2}, k}=(-1)^{m_{1}+n_{2}} h_{l, n_{2}, n_{1}, m_{2}, m_{1},-k},
$$

where the bar stands for complex conjugation.

\section{NORMAL FORM AT HIGHER ORDER}

The Hamiltonian with the quadratic part given by (7) is suitable to start the normal form process. Actually we carry out this process up to any (high) order. This computation is based on the following

Proposition 2. Let us assume that the frequencies $\omega_{0}$ and $\omega_{1}$ are rationally independendent, that is, $\boldsymbol{\omega} \cdot \boldsymbol{k}=k_{0} \omega_{0}+k_{1} \omega_{1} \neq 0$, for all $k_{0}$, $k_{1} \in Z-\{0\}$; then the (formal) normal form $\Lambda$ of the Hamiltonian $H$, in the complex variables introduced in the last section, takes the form

$\Lambda(\theta, q, I, p)=\omega_{0} I+i \omega_{1}\left(q_{1} p_{1}+q_{2} p_{2}\right)+q_{2} p_{1}+\mathcal{N}\left(I, q_{1} p_{2}, i\left(q_{1} p_{1}+q_{2} p_{2}\right)\right)$,

where $\mathcal{N}(A, B, C)$ is a real formal power series in the variables $(A, B, C)$ which begins with terms of second degree.

Proof. The proof of this proposition uses the Lie series method to remove in an increasing order the "non-resonant terms" of the Hamiltonian. The sketch of the proof consists of the following process:

1. The homological equation to be solved in any step of the normal form process takes the form:

$$
\left\{H_{2}, G\right\}+R=N,
$$

where $R$ contains the terms to be removed (of a given degree $s$ ) by a suitable $G$, while $N$ stands for the non-removable terms.

2. If $\mathrm{F}$ is a monomial like $F=a_{l, m, n, k} e^{i k \theta} I^{l} q_{1}^{m_{1}} q_{2}^{m_{2}} p_{1}^{n_{1}} p_{2}^{n_{2}}$, then

$$
L_{H_{2}} F:=-\left\{H_{2}, F\right\}=\left(\Omega_{m_{1}, m_{2}, n_{1}, n_{2}, k}+m_{1} \frac{q_{2}}{q_{1}}-n_{2} \frac{p_{1}}{p_{2}}\right) F,
$$

where we have defined

$$
\Omega_{m, n, k}=\Omega_{m_{1}, m_{2}, n_{1}, n_{2}, k}:=i \omega_{0} k+i \omega_{1}\left(m_{1}+m_{2}-n_{1}-n_{2}\right) .
$$


As in addition, the frequencies $\omega_{0}$ and $\omega_{1}$ are rationally independent, it can be shown that the necessary conditions for a monomial to be resonant are

$$
k=0 \text { and }\left(\Omega_{m, n, 0}=0 \Leftrightarrow m_{1}+m_{2}=n_{1}+n_{2}\right) .
$$

By discussing the structure of the equation (11), we can see that if the above resonance conditions are not fulfilled by the monomial $\mathrm{F}$, one can remove the corresponding term in the homological equations giving the adequate value to the coefficients $a_{l, m, n, k}$ of the generating function $G$.

3. By a more careful analysis of the algebraic structure of equation (11), one can see that the possible non-removable terms in the normal form are the ones given by

$$
f=\sum_{l, M} \sum_{0 \leq m_{1}, n_{2} \leq M} f_{l, m_{1}, M-m_{1}, M-n_{2}, n_{2}} I^{l} q_{1}^{m_{1}} q_{2}^{M-m_{1}} p_{1}^{M-n_{2}} p_{2}^{n_{2}} .
$$

Introducing the new variables,

$$
\left.\begin{array}{ll}
X=q_{1} p_{2}, & Y=q_{2} p_{1} \\
Q=i\left(q_{1} p_{1}+q_{2} p_{2}\right), & P=\left(q_{1} p_{1}-q_{2} p_{2}\right)
\end{array}\right\}
$$

(note that $X, Y, Q$ and $P$ are real variables), it can be proved (see [12] for details) that the terms in (15) which cannot be removed, written in the variables (16) are

$$
\sum_{l, M} \sum_{n=0}^{M} g_{l, n, M-n} I^{l} X^{n} Q^{M-n}
$$

with $g_{l, n, M-n} \in R$. Thus, the normal form is a formal power series in the variables $I, q_{1} p_{2}$ and $i\left(q_{1} p_{1}+q_{2} p_{2}\right)$, with real coefficients.

\section{THE RESONANT NORMAL FORM}

As stated in proposition 2 the resonant normal form around the critical periodic orbit is given by (10) where $\mathcal{N}(A, B, C)$ is a real formal power series expansion, beginning at degree 2 with respect to $(A, B, C)$. Nevertheless, in what follows we will discuss the properties of $\mathcal{N}$ as if it were a real analytic function, defined around $A=B=C=0$. In fact, if Diophantine conditions are assumed, this is so if $\mathcal{N}$ is truncated at some finite order, as usual in practical computations involving normal forms.

In order to simplify the Hamiltonian equations associated to $\Lambda$, we will introduce the following system of canonical coordinates $\left(r, p_{r}, \varphi, p_{\varphi}\right)$, given 
by

$$
\begin{aligned}
& q_{1}=\sqrt{r} \exp (i \varphi) \\
& q_{2}=\sqrt{r} \exp (i \varphi) p_{r}-\frac{\exp (i \varphi)}{2 i \sqrt{r}} p_{\varphi}, \\
& p_{1}=\sqrt{r} \exp (-i \varphi) p_{r}+\frac{\exp (-i \varphi)}{2 i \sqrt{r}} p_{\varphi}, \\
& p_{2}=-\sqrt{r} \exp (-i \varphi),
\end{aligned}
$$

where $\varphi \in S^{1}$, and where we remark that $r>0, p_{r}$ and $p_{\varphi}$ are real variables. We can write them as:

$$
\begin{aligned}
r & =-q_{1} p_{2}, \\
p_{r} & =\frac{q_{1} p_{1}-q_{2} p_{2}}{2 r}, \\
p_{\varphi} & =i\left(q_{1} p_{1}+q_{2} p_{2}\right) .
\end{aligned}
$$

The expression of $\Lambda$ in these new coordinates becomes (we denote it by $\Lambda$ again)

$$
\Lambda\left(\theta, \varphi, r, I, p_{\varphi}, p_{r}\right)=\omega_{0} I+\omega_{1} p_{\varphi}+r p_{r}^{2}+\frac{p_{\varphi}^{2}}{4 r}+\mathcal{N}\left(I,-r, p_{\varphi}\right) .
$$

The corresponding Hamiltonian equations are:

$$
\begin{aligned}
\dot{\theta} & =\omega_{0}+\partial_{1} \mathcal{N}\left(I,-r, p_{\varphi}\right) \\
\dot{\varphi} & =\omega_{1}+\frac{p_{\varphi}}{2 r}+\partial_{3} \mathcal{N}\left(I,-r, p_{\varphi}\right), \\
\dot{r} & =2 r p_{r} \\
\dot{I} & =0 \\
\dot{p}_{\varphi} & =0 \\
\dot{p}_{r} & =-p_{r}^{2}+\frac{p_{\varphi}^{2}}{4 r^{2}}+\partial_{2} \mathcal{N}\left(I,-r, p_{\varphi}\right) .
\end{aligned}
$$

We have that $I$ and $p_{\varphi}$ are first integrals of the differential equations associated to the resonant normal form. Hence, by taking fixed values of $I \equiv I^{0}$ and $p_{\varphi} \equiv p_{\varphi}^{0}$, we can reduce the $\Lambda$ to a one degree-of-freedom Hamiltonian system, given by

$$
\Lambda_{0}\left(r, p_{r}\right)=r p_{r}^{2}+\frac{\left(p_{\varphi}^{0}\right)^{2}}{4 r}+\mathcal{N}\left(I^{0},-r, p_{\varphi}^{0}\right)
$$

We can compute: 
(i) 2-dimensional tori of $\Lambda$ as fixed points of $\Lambda_{0}$.

(ii) 3-dimensional tori of $\Lambda$ as periodic orbits of $\Lambda_{0}$.

We will focus our attention on the 2-D tori of $\Lambda_{0}$. The equilibrium points of such reduced system are given by $p_{r}=0$, and $r$ as a solution of

$$
\frac{\left(p_{\varphi}^{0}\right)^{2}}{4 r^{2}}+\partial_{2} \mathcal{N}\left(I^{0},-r, p_{\varphi}^{0}\right)=0 .
$$

In order to parametrize the solutions of (18), we introduce a new (dependent) variable $N$ defined as

$$
N=\partial_{2} \mathcal{N}\left(I,-r, p_{\varphi}\right)
$$

and from equation (18) we have to restrict $N \leq 0$ and $r \geq 0$. Thus, we can parametrize the solutions of (18) as

$$
\begin{aligned}
p_{\varphi}^{ \pm}(r, N) & = \pm 2 r \sqrt{-N} \\
p_{r}^{ \pm}(r, N) & =0 \\
N & =\partial_{2} \mathcal{N}\left(I^{ \pm}(r, N),-r, \pm 2 r \sqrt{-N}\right)
\end{aligned}
$$

where $I^{ \pm}$as function of $(r, N)$ are obtained from the implicit function theorem if we assume that

$$
\partial_{2,1} \mathcal{N}(0,0,0) \neq 0 .
$$

Remark 3. It seems as if we had two different families of fixed points of $\Lambda_{0}$ from (20), and hence, two different families of "bifurcated" 2-dimensional tori from $\Lambda$, but we remark that when we put $N=0$, then $I^{+}(r, 0)=$ $I^{-}(r, 0)$. In fact the sign \pm is only associated to the choice of the sign of $p_{\varphi}$ in (19).

Finally, the frequencies of the quasi-periodic motion on the 2-dimensional tori computed are given by

$$
\begin{aligned}
\dot{\theta} & =\Omega_{0}^{ \pm} \equiv \omega_{0}+\partial_{1} \mathcal{N}\left(I^{ \pm}(r, N),-r, \pm 2 r \sqrt{-N}\right) \\
\dot{\varphi} & =\Omega_{1}^{ \pm} \equiv \omega_{1} \pm \sqrt{-N}+\partial_{3} \mathcal{N}\left(I^{ \pm}(r, N),-r, \pm 2 r \sqrt{-N}\right)
\end{aligned}
$$




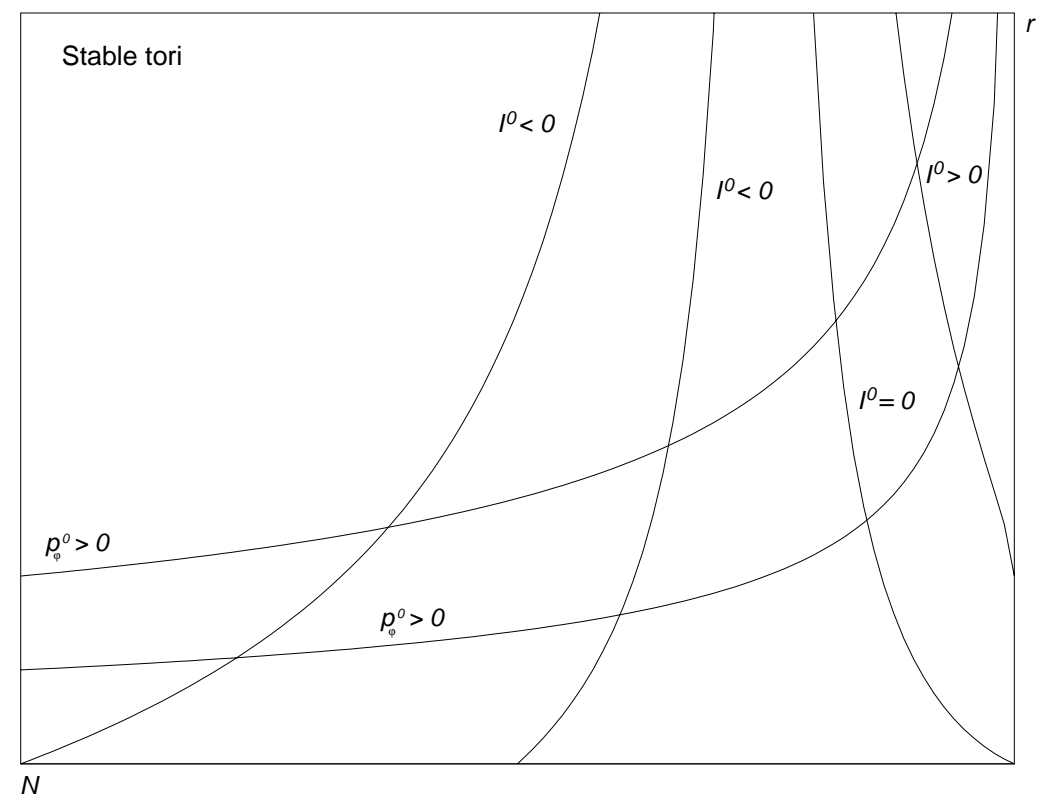

FIG. 1. Direct case. The stable tori unfold on the unstable side. The hyperbolas obtained from (19) and (20) show families of $2 \mathrm{D}$ tori with a fixed value of $p_{\varphi}=p_{\varphi}^{0}\left(p_{\varphi}^{0}>0\right.$ in this plot $)$, whereas each "vertical" curve corresponds to a fixed $I=I^{0}$. 


\section{UNFOLDING AND STABILITY OF THE BIFURCATED 2-DIMENSIONAL TORI}

The eigenvalues of the linearized system of $\Lambda_{0}$ around the equilibrium points computed before are:

$$
\pm \sqrt{4 N-2 r \partial_{2,2} \mathcal{N}\left(I^{ \pm}(r, N),-r, \pm 2 r \sqrt{-N}\right)} .
$$

so two cases have to be distinguished:

- If $\partial_{2,2} \mathcal{N}(0,0,0)>0$, then the eigenvalues are purely imaginary for small values of $r \geq 0$ and $N \leq 0$, both not simultaneously zero (case of a direct bifurcation).

- If $\partial_{2,2} \mathcal{N}(0,0,0)<0$, then we have a transition from stability to instability characterized by:

$$
4 N-2 r \partial_{2,2} \mathcal{N}\left(I^{ \pm}(r, N),-r, \pm 2 r \sqrt{-N}\right)=0,
$$

(case of an inverse bifurcation). This expression allows to write $r$ as function of $N$ :

$$
r \equiv r^{ \pm}(N), \text { with } r^{ \pm}(0)=0 .
$$

Both cases are illustrated in figures 1 and 2. The plots therein are obtained taking only the terms of degree two in $\mathcal{N}$ (fourth order in the Hamiltonian). In both figures, the vertical axis corresponds to $p_{\varphi}=0$ (stable tori in the direct bifurcation and unstable in the inverse case); and the horizontal axis contains the periodic orbits, $r=0$ (stable if $I<0$ and unstable if $I>0$.

Remark 4. Of course, the dynamics described in the last two sections corresponds to the dynamics of the truncated normal form, that is with $\mathcal{N}\left(I,-r, p_{\varphi}\right)$ as a polynomial of finite (arbitrarily high) degreee. Actually the Hamiltonian consists of this truncated normal form plus a remainder. Bounds on this remainder as well as the existence of the bifurcated 2D-tori (in Cantor sets) for the complete (non-integrable) Hamiltonian wil be the purpose of a future paper ([12]).

\section{ACKNOWLEDGMENT}

The first author acknowledges the Departament de Matemàtiques (Universitat Autònoma de Barcelona) and the Centre de Recerca Matemàtica (Bellaterra) where this research has been carried out. This work has been partially supported by the Catalan grant CIRIT number 2000SGR-00027, by the Spanish grant number BFM2000-0623 and by the INTAS grant $00-221$. 


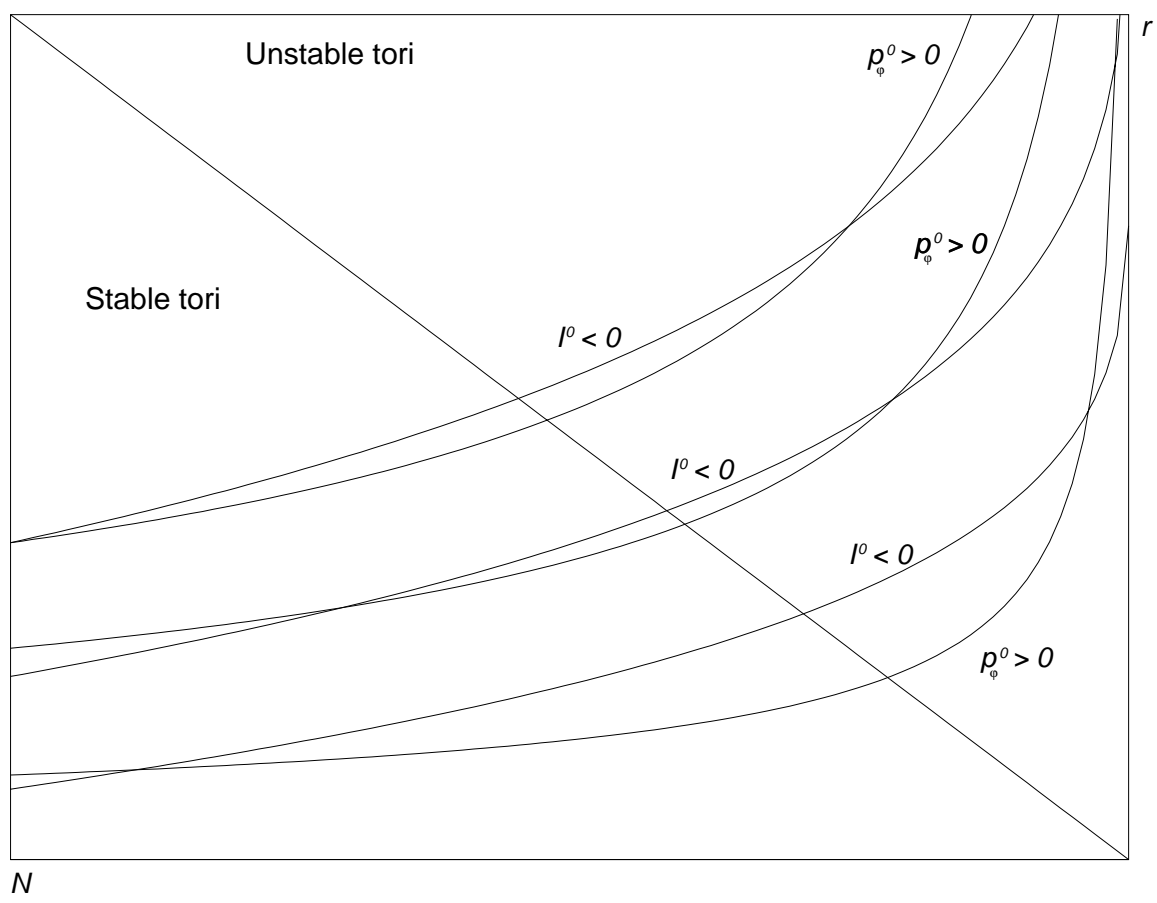

FIG. 2. Inverse case. The unstable tori unfold on the stable side. The curves represent families of $2 \mathrm{D}$-tori for different values of $p_{\varphi}=p_{\varphi}^{0}\left(p_{\varphi}^{0}>0\right.$ in this plot $)$ and $I=I^{0}$. The "transversal line" separates the unstable an stable tori region. 


\section{REFERENCES}

1. T. J. BRIDGES, Stability of periodic solutions near a colision of eigenvalues of opposite signature, Math. Proc.Camb. Phil. Soc. 109 (1991), 375-403.

2. T. J. BRIDges AND R. H. CuShMAN, Unipotent normal forms for symplectic mappings, Physica D 65 (1993), 211-241.

3. T. J. Bridges, R. H. Cushman And R. S. Mackay, Dynamics near an irrational collision of eigenvalues for symplectic mappings, Fields Institute Communications 4 (1995), 61-79.

4. G. Contopoulos And A. Giorgilli, Bifurcations and complex instability in a 4dimensional symplectic mapping, Meccanica 23 (1988), 19-28.

5. G. Contopoulos And B. Barbanis, Periodic orbits and their bifurcations in a-3D system, Cel. Mech. Dynam. Astron. 59 (1994), 279-300.

6. D. G. HegGie, Bifurcation at complex instability, Cel. Mech. 35 (1985), 357-382.

7. A. JoRba AND J. VillanueVA, Numerical computation of normal forms and some periodic orbits of the RTBP, Physica D 114 (1998), 197-229.

8. M. Ollé And D. Pfenniger, Bifurcation at complex instability, in Hamiltonian systems with three or more degrees of freedom, C. Simó (Ed.), NATO Adv. Sci. Inst. Ser. C Math. Phys. Sci. 53, Kluwer Acad. Publ. Dordrecht, Holland, 2000, p. $518-522$.

9. M. Ollé And D. Pfenniger, Vertical orbital structure and the lagrangian points in barred galaxies, Astron. Astrophys. 334 (1998), 829-839.

10. M. OllÉ AND J. R. PACHA, Dynamics and bifurcation near the transition from stability to complex instability: some examples, in Actas del XV C.E.D.Y.A., V C.M.A. 1, Ed. Servicio de Publicacions da Universidades de Vigo, 1998, p. 339-344.

11. M. OllÉ AND J. R. PACHA, The 3D Elliptic RTBP: periodic orbits which bifurcate from limiting restricted problems: complex instability, Astron. Astrophys 351 (1999), 1149-1164.

12. M. Ollé, J. R. Pacha and J. Villanueva, in preparation

13. D. Pfenniger, Numerical study of complex instability. I Mappings, Astron. Astrophys. 150 (1985), 97-111.

14. D. Pfenniger, Numerical study of complex instability. II Barred galaxy bulges, Astron. Astrophys. 150 (1985), 112-128.

15. D. Pfenniger, Stability of the lagrangian points in stellar bars, Astron. Astrophys. 230 (1990), 55-66.

16. J. VAN DER MEER, The Hamiltonian Hopf bifurcation, Lecture Notes in Maths., 1160 (1985), Springer-Verlag. 